\title{
Performance Analysis and Optimization of an Adaptive Admission Control Scheme in Cognitive Radio Networks
}

\author{
Shunfu Jin, ${ }^{1,2}$ Yuan Zhao, ${ }^{1,2}$ Wuyi Yue, ${ }^{3}$ and Zsolt Saffer ${ }^{4}$ \\ ${ }^{1}$ College of Information Science and Engineering, Yanshan University, Qinhuangdao 066004, China \\ ${ }^{2}$ Key Laboratory for Computer Virtual Technology and System Integration of Hebei Province, Yanshan University, \\ Qinhuangdao 066004, China \\ ${ }^{3}$ Department of Intelligence and Informatics, Konan University, Kobe 658-8501, Japan \\ ${ }^{4}$ Department of Networked Systems and Services, Budapest University of Technology and Economics, Budapest 1521, Hungary
}

Correspondence should be addressed to Wuyi Yue; yue@konan-u.ac.jp

Received 1 August 2013; Revised 6 October 2013; Accepted 10 October 2013

Academic Editor: Pui-Sze Chow

Copyright (C) 2013 Shunfu Jin et al. This is an open access article distributed under the Creative Commons Attribution License, which permits unrestricted use, distribution, and reproduction in any medium, provided the original work is properly cited.

In cognitive radio networks, if all the secondary user (SU) packets join the system without any restrictions, the average latency of the SU packets will be greater, especially when the traffic load of the system is higher. For this, we propose an adaptive admission control scheme with a system access probability for the SU packets in this paper. We suppose the system access probability is inversely proportional to the total number of packets in the system and introduce an Adaptive Factor to adjust the system access probability. Accordingly, we build a discrete-time preemptive queueing model with adjustable joining rate. In order to obtain the steady-state distribution of the queueing model exactly, we construct a two-dimensional Markov chain. Moreover, we derive the formulas for the blocking rate, the throughput, and the average latency of the SU packets. Afterwards, we provide numerical results to investigate the influence of the Adaptive Factor on different performance measures. We also give the individually optimal strategy and the socially optimal strategy from the standpoints of the SU packets. Finally, we provide a pricing mechanism to coordinate the two optimal strategies.

\section{Introduction}

Nowadays, radio spectrum is one of the scarcest and most invaluable resources for wireless communications [1]. However, actual measures show that the utilization of the spectrum is very low in practical networks [2]. This underutilization of the spectrum motivated the researchers to explore how to improve the allocation of the spectrum. As a result of this tendency, cognitive radio networks have emerged as a promising technology for solving the problem of the spectrum underutilization [3].

There are two types of users in cognitive radio networks, namely, Primary Users (PUs) and Secondary Users (SUs) [4]. The PUs access the licensed spectrum with a preemptive priority. The SUs can make opportunistic use of the licensed spectrum whenever the spectrum is not occupied by the PUs.

In recent years, research interest in cognitive radio networks has grown rapidly, and a great amount of research has dealt with the system performance of cognitive radio networks [5].

In [6], Su and Zhang developed a Markov chain to obtain the aggregate throughput of the SUs with two channel sensing policies. In [7], Kim et al. analyzed a carrier sense multiple access strategy in multichannel cognitive radio networks with a three-dimensional Markov chain. They derived the throughput and the packet delay of the SUs. Moreover, priority queueing systems have been widely adopted in performance studies of cognitive radio networks, since the priority queueing systems are suitable to model the nonidentical behaviors of different types of customers that join the systems [8]. In [9], Do et al. applied an M/G/1 preemptive priority queueing scheme to analyze the average waiting time of SU packets in the system. In [10], Smith et al. considered an $\mathrm{M} / \mathrm{M} / \mathrm{N} / \mathrm{N}$ preemptive priority queue with two types of customers. They investigated the mean number and the 
blocking probabilities for both kinds of users in cognitive radio networks.

The above mentioned research works on cognitive radio networks have focused on the system access strategy with 1 persistent scheme for the SUs. That is to say, the SU packets are supposed to join the system no matter how many packets are available in the system. Obviously, this kind of system access strategy will lead to a greater latency of the SUs. In networks, greater latency (also called time delay) may be potential source of poor performance, even of instability [11-13]. In order to control the access of SU packets in cognitive radio networks, in [14], Li and Han proposed an access threshold for the SU packets. They assumed the SU packets would not join the system when the queue length of the SU packets was equal to or greater than the access threshold. They supposed that the queue length could be received in a broadcast message sent out from the base station. With numerical results, they gave the individually and socially optimal access thresholds for the SU packets. However, the observable queue assumption will increase the system overhead due to the necessity of a signaling scheme. In [15], Turhan et al. assumed that a newly arriving SU packet would be admitted to join the system only when the total number of packets in the system was smaller than an access threshold. One drawback of the models in $[14,15]$ is that the access threshold was fixed and a newly arriving SU packet would definitely join the system when the number of packets did not exceed the access threshold. For better performance, we should adjust the system access of a newly arriving SU packet adaptively according to the traffic load of the system.

On the other hand, most of the researches on the performance evaluation of cognitive radio networks was performed in continuous-time domain. However, as nowadays communication systems are digital [16], it would be more suitable to use discrete-time system models rather than their continuous-time counterparts when analyzing or designing digital transmitting systems [17, 18].

In this paper, in order to control the system access of SU packets adaptively, we propose an admission control scheme with a system access probability. By introducing an Adaptive Factor, we assume the system access probability for a newly arriving SU packet is inversely proportional to the total number of packets in the system. We call this admission control scheme an adaptive admission control scheme. Based on the working principle of the adaptive admission control scheme, by considering the digital nature of modern communication, we build a discrete-time preemptive queueing model with priority and adjustable joining rate. We exactly evaluate the system performance by examining the blocking rate, the throughput, and the average latency of the SU packets. Furthermore, we compare the individually optimal strategy and the socially optimal strategy for the SU packets and propose a pricing mechanism for the SU packets.

The remainder of this paper is organized as follows. An adaptive admission control scheme for the SU packets and the system model are proposed in Section 2. The performance analysis is carried out in Section 3. In Section 4, the formulas for the performance measures, such as the blocking rate, the throughput and the average latency of the SU packets are obtained. Moreover, numerical results are provided to show the influence of the Adaptive Factor on different performance measures. In Section 5, the individually optimal strategy and the socially optimal strategy for the SU packets are compared, and a pricing mechanism is proposed to coordinate the two optimal strategies. Finally, conclusions are drawn in Section 6.

\section{System Model for an Adaptive Admission Control Scheme}

2.1. An Adaptive Admission Control Scheme in Cognitive Radio Networks. In this paper, we consider one licensed spectrum with a single channel in a kind of centralized cognitive radio network, in which there is a central controller that can allocate the spectrum for the PUs and the SUs in the network. The PU packets have preemptive priority to occupy the channel, and the SU packets can only make opportunistic use of the channel. Obviously, the greater the number of SU packets in the system is, the longer the latency of an SU packet and the higher the administration cost will be. Therefore, we propose an adaptive admission control scheme for the SU packets. The working principle for the adaptive admission control scheme is illustrated in Figure 1.

In Figure 1, there are five points to be emphasized as follows.

(1) In order to decrease the latency of the SU packets, a finite buffer with capacity of $K(K>0)$ is set for the SU packets. On the other hand, to satisfy the latency requirement of the PUs to a maximum extend, no buffer is prepared for the PUs.

(2) In the adaptive admission control scheme, the central controller counts the packets in the system periodically. When a new SU packet arrives at the system, the central controller will admit this SU packet with probability $\beta_{i}=1 /(\alpha i+1)$ or refuse this SU packet with probability $\left(1-\beta_{i}\right)$, where $i$ is the number of packets in the system and $\alpha$ is the Adaptive Factor. We call $\beta_{i}$ the system access probability, which is inversely proportional to the number of packets in the system.

(3) When an SU packet is admitted to join the system, if the channel is being occupied by another packet (a PU packet or an SU packet), this SU packet will queue in the buffer. If the buffer is full, this SU packet will be blocked.

(4) When a PU packet joins the system, if the channel is idle, this PU packet will definitely occupy the channel directly; if the channel is being occupied by another PU packet, the newly arriving PU packet will be blocked; if the channel is being occupied by an SU packet, the newly arriving PU packet will interrupt the transmission of this SU packet and occupy the channel immediately.

(5) When the transmission of an SU packet is interrupted, this SU packet will return back to the buffer of the SUs and is queued at the head. If the buffer of the SUs is full, the SU packet queued at the end will be 


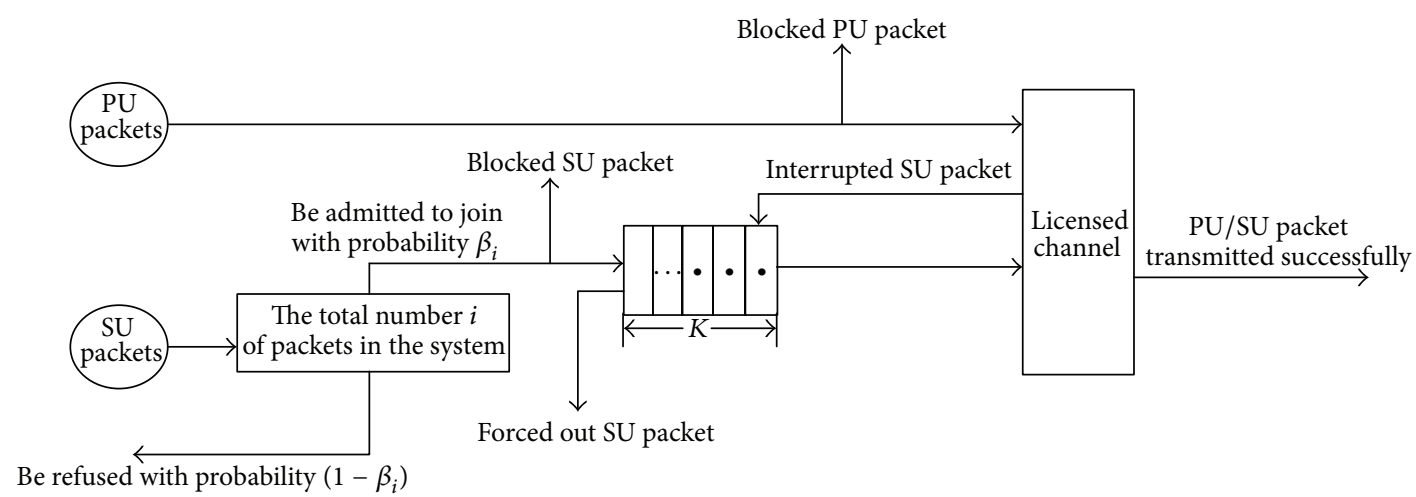

FIgURE 1: Adaptive admission control scheme in cognitive radio networks.

forced to leave the system. If the new admission of an SU packet and the interruption of an SU packet occur simultaneously, then due to having only one vacancy in the buffer, the interrupted SU packet will join the system, and the newly admitted SU packet will be blocked by the system. Hence, the interrupted $\mathrm{SU}$ packet is assumed to have a higher priority than the newly admitted SU packet.

From the above description, it is clear that the system access probability is dependent on the total number of packets in the system. The larger the number of packets in the system is, the less the possibility that a newly arriving SU packet will be admitted to join the system. On the other hand, the Adaptive Factor can adjust the impact of the total number of SU packets on the system access probability. Obviously, this adaptive admission control scheme can reduce the average latency of the SU packets.

2.2. System Model. Based on the adaptive admission control scheme proposed in this paper, a preemptive priority queueing model with adjustable joining rate can be built.

To capture the digital nature of modern networks, we consider the queueing model in discrete-time field. The time axis is divided into slots with equal length. The slot boundaries are marked as $n(n=1,2, \ldots)$. The arrivals of the packets can occur immediately after the beginning instant of a slot, and the departures of the packets can occur just prior to the end of a slot. Taking the instant $t=n$ as an example, the arrival of a packet is supposed to occur at $\left(n, n^{+}\right)$, and the departure of a packet is supposed to occur at $\left(n^{-}, n\right)$. That is to say, an early arrival system (EAS) is considered. In order to avoid ambiguity, we assume that a new SU packet arriving during the interval of $\left((n+1),(n+1)^{+}\right)$will be admitted or refused by the system based on the number of packets in the system at the instant $t=n^{+}$.

The interarrival times and transmission times for both the two kinds of packets (PU packets and SU packets) are assumed to be independent, identically distributed (i.i.d) random variables following geometrical distributions [14]. The arriving intervals of the PU packets and the SU packets are supposed to follow geometrical distributions with parameters $\lambda_{1}\left(0<\lambda_{1}<1, \bar{\lambda}_{1}=1-\lambda_{1}\right)$ and $\lambda_{2}\left(0<\lambda_{2}<1, \bar{\lambda}_{2}=1-\lambda_{2}\right)$, respectively. According to the adaptive admission control scheme, when the total number of packets in the system is $i$, we denote the actual joining rate $\gamma_{i}$ of the SU packets that are admitted to join the system as $\gamma_{i}=\lambda_{2} /(\alpha i+1)$, where $\alpha$ is the Adaptive Factor. The transmission times of the PU packets and the SU packets are assumed to follow geometrical distributions with parameters $\mu_{1}\left(0<\mu_{1}<1, \bar{\mu}_{1}=1-\mu_{1}\right)$ and $\mu_{2}\left(0<\mu_{2}<1, \bar{\mu}_{2}=1-\mu_{2}\right)$, respectively. The traffic intensity of the PU packets and the SU packets are defined as $\rho_{1}\left(\rho_{1}=\lambda_{1} / \mu_{1}\right)$ and $\rho_{2}\left(\rho_{2}=\lambda_{2} / \mu_{2}\right)$, respectively.

Let $L_{n}=i(i=0,1,2, \ldots, K+1)$ be the total number of packets in the system at the instant $t=n^{+}$, and let $L_{n}^{(1)}=$ $j(j=0,1)$ be the number of PU packets in the system at the instant $t=n^{+} .\left\{L_{n}, L_{n}^{(1)}\right\}$ constitutes a two-dimensional Markov chain [13]. The state spaces of this Markov chain are given as follows:

$$
\boldsymbol{\Omega}=(0,0) \cup\{(i, j): 1 \leq i \leq K+1, j=0,1\},
$$

where state $(0,0)$ denotes there is no packet in the system; state $(i, 0)$ denotes that there are $i$ SU packets and no PU packet in the system; state $(i, 1)$ denotes that there are $(i-1)$ SU packets and one PU packet in the system.

\section{Performance Analysis}

We define the system phase as the total number of packets in the system. Let $\mathbf{Q}$ be the state transition probability matrix for the system phases. $\mathbf{Q}$ can be given as a $(K+2) \times(K+2)$ block-structured matrix as follows:

$$
\mathbf{Q}=\left(\begin{array}{cccccc}
\mathbf{Q}_{0,0} & \mathbf{Q}_{0,1} & \mathbf{Q}_{0,2} & & & \\
\mathbf{Q}_{1,0} & \mathbf{Q}_{1,1} & \mathbf{Q}_{1,2} & \mathbf{Q}_{1,3} & & 0 \\
& \mathbf{Q}_{2,1} & \mathbf{Q}_{2,2} & \mathbf{Q}_{2,3} & \mathbf{Q}_{2,4} & \\
& \ddots & \ddots & \ddots & \ddots & \\
0 & & & \mathbf{Q}_{K, K-1} & \mathbf{Q}_{K, K} & \mathbf{Q}_{K, K+1} \\
& & & & \mathbf{Q}_{K+1, K} & \mathbf{Q}_{K+1, K+1}
\end{array}\right),
$$

where $\mathbf{Q}_{u, v}$ is the transition probability matrix from the system phase $u$ to the system phase $v, u=0,1, \ldots, K+1$, $v=0,1, \ldots, K+1$.

Q can be discussed according to different system phases as follows. 
(1) At the instant $t=n^{+}$, if the system phase $u=0$, that is, there is no packet in the system, the system phase will be $v(v=0,1,2)$ at the instant $t=(n+1)^{+}$.

For the system phase $v=0$, namely, there is no packet in the system at the instant $t=(n+1)^{+}$, the transition probability matrix $\mathbf{Q}_{0,0}$ is in fact a vector with only one scalar value given as follows:

$$
\mathbf{Q}_{0,0}=\bar{\lambda}_{1} \bar{\lambda}_{2}
$$

For the system phase $v=1$, namely, there is one SU packet or one PU packet in the system at the instant $t=(n+1)^{+}$, the transition probability matrix $\mathbf{Q}_{0,1}$ is a row vector with two elements given as follows:

$$
\mathbf{Q}_{0,1}=\left(\bar{\lambda}_{1} \lambda_{2}, \lambda_{1} \bar{\lambda}_{2}\right)
$$

For the system phase $v=2$, namely, there is one SU packet and one PU packet in the system at the instant $t=(n+1)^{+}$, the transition probability matrix $\mathbf{Q}_{0,2}$ is a row vector with two elements given as follows:

$$
\mathbf{Q}_{0,2}=\left(0, \lambda_{1} \lambda_{2}\right)
$$

(2) At the instant $t=n^{+}$, if the system phase $u=1$, that is, there is one packet in the system, the system phase will be $v(v=0,1,2,3)$ at the instant $t=(n+1)^{+}$.

The system phase $v=0$ means there is no packet in the system at the instant $t=(n+1)^{+}$. For this case, the packet (one PU packet or one SU packet) in the system leaves, and there is no packet arrival. So, the transition probability matrix $\mathbf{Q}_{1,0}$ is a column vector with two elements given as follows:

$$
\mathbf{Q}_{1,0}=\left(\begin{array}{l}
\bar{\lambda}_{1}\left(1-\gamma_{1}\right) \mu_{2} \\
\bar{\lambda}_{1}\left(1-\gamma_{1}\right) \mu_{1}
\end{array}\right) \text {. }
$$

The system phase $v=1$ means there is one packet in the system at the instant $t=(n+1)^{+}$. For this case, either the packet (one PU packet or one SU packet) in the system does not leave, and there is no packet arrival, or the PU packet in the system does not leave, and there is one PU packet arrival, or the packet (one PU packet or one SU packet) in the system leaves, and there is one packet (one PU packet or one SU packet) arrival. So, the transition probability matrix $\mathbf{Q}_{1,1}$ can be given as follows:

$$
\mathbf{Q}_{1,1}=\left(\begin{array}{cc}
\bar{\lambda}_{1}\left(\left(1-\gamma_{1}\right) \bar{\mu}_{2}+\gamma_{1} \mu_{2}\right) & \lambda_{1}\left(1-\gamma_{1}\right) \mu_{2} \\
\bar{\lambda}_{1} \gamma_{1} \mu_{1} & \left(1-\gamma_{1}\right)\left(\lambda_{1} \mu_{1}+\bar{\mu}_{1}\right)
\end{array}\right) .
$$

The system phase $v=2$ means there are two packets in the system at the instant $t=(n+1)^{+}$. For this case, either the SU packet in the system does not leave, and there is one packet (one PU packet or one SU packet) arrival, or the PU packet in the system does not leave, and there are two packet (one PU packet and one SU packet) arrivals, or the PU packet in the system does not leave, and there is one SU packet arrival, or the packet (one PU packet or one SU packet) in the system leaves, and there are two packet (one PU packet and one SU packet) arrivals. So, the transition probability matrix $\mathbf{Q}_{1,2}$ can be given as follows:

$$
\mathbf{Q}_{1,2}=\left(\begin{array}{cc}
\bar{\lambda}_{1} \gamma_{1} \bar{\mu}_{2} & \lambda_{1}\left(\left(1-\gamma_{1}\right) \bar{\mu}_{2}+\gamma_{1} \mu_{2}\right) \\
0 & \gamma_{1}\left(\lambda_{1} \mu_{1}+\bar{\mu}_{1}\right)
\end{array}\right) .
$$

The system phase $v=3$ means there are three packets in the system at the instant $t=(n+1)^{+}$. For this case, the SU packet in the system does not leave, and there are two packet (one PU packet and one SU packet) arrivals. So, the transition probability matrix $\mathbf{Q}_{1,3}$ can be given as follows:

$$
\mathbf{Q}_{1,3}=\left(\begin{array}{cc}
0 & \lambda_{1} \gamma_{1} \bar{\mu}_{2} \\
0 & 0
\end{array}\right)
$$

(3) At the instant $t=n^{+}$, if the system phase $2 \leq u \leq K-1$, that is, there are $u(2 \leq u \leq K-1)$ packets in the system, the system phase will be $v(v=u-1, u, u+$ $1, u+2)$ at the instant $t=(n+1)^{+}$.

The system phase $v=u-1$ means there are $(u-1)$ packets in the system at the instant $t=(n+1)^{+}$. For this case, one of the packets in the system leaves, and there is no packet arrival. So the transition probability matrix $\mathbf{Q}_{u, u-1}$ can be given as follows:

$$
\mathbf{Q}_{u, u-1}=\left(\begin{array}{ll}
\bar{\lambda}_{1}\left(1-\gamma_{u}\right) \mu_{2} & 0 \\
\bar{\lambda}_{1}\left(1-\gamma_{u}\right) \mu_{1} & 0
\end{array}\right) .
$$

Similar to the matrix structures shown in (7)-(9), the transition probability matrix $\mathbf{Q}_{u, v}$ for $v(v=u, u+1, u+2)$ can be given as follows:

$$
\begin{aligned}
& \mathbf{Q}_{u, u}=\left(\begin{array}{cc}
\bar{\lambda}_{1}\left(\left(1-\gamma_{u}\right) \bar{\mu}_{2}+\gamma_{u} \mu_{2}\right) & \lambda_{1}\left(1-\gamma_{u}\right) \mu_{2} \\
\bar{\lambda}_{1} \gamma_{u} \mu_{1} & \left(1-\gamma_{u}\right)\left(\lambda_{1} \mu_{1}+\bar{\mu}_{1}\right)
\end{array}\right), \\
& \mathbf{Q}_{u, u+1}=\left(\begin{array}{cc}
\bar{\lambda}_{1} \gamma_{u} \bar{\mu}_{2} & \lambda_{1}\left(\left(1-\gamma_{u}\right) \bar{\mu}_{2}+\gamma_{u} \mu_{2}\right) \\
0 & \gamma_{u}\left(\lambda_{1} \mu_{1}+\bar{\mu}_{1}\right)
\end{array}\right) \\
& \mathbf{Q}_{u, u+2}=\left(\begin{array}{cc}
0 & \lambda_{1} \gamma_{u} \bar{\mu}_{2} \\
0 & 0
\end{array}\right) \text {. }
\end{aligned}
$$

(4) At the instant $t=n^{+}$, if the system phase $u=K$, that is, there is only one vacancy in the buffer, the system phase will be $v(v=K-1, K, K+1)$ at the instant $t=(n+1)^{+}$.

Similar to the matrix structures shown in (10) and (11), the transition probability matrix $\mathbf{Q}_{K, K-1}$ and $\mathbf{Q}_{K, K}$ can be given as follows:

$$
\begin{gathered}
\mathbf{Q}_{K, K-1}=\left(\begin{array}{cc}
\bar{\lambda}_{1}\left(1-\gamma_{K}\right) \mu_{2} & 0 \\
\bar{\lambda}_{1}\left(1-\gamma_{K}\right) \mu_{1} & 0
\end{array}\right) \\
\mathbf{Q}_{K, K}=\left(\begin{array}{cc}
\bar{\lambda}_{1}\left(\left(1-\gamma_{K}\right) \bar{\mu}_{2}+\gamma_{K} \mu_{2}\right) & \lambda_{1}\left(1-\gamma_{K}\right) \mu_{2} \\
\bar{\lambda}_{1} \gamma_{K} \mu_{1} & \left(1-\gamma_{K}\right)\left(\lambda_{1} \mu_{1}+\bar{\mu}_{1}\right)
\end{array}\right) .
\end{gathered}
$$


The system phase $v=K+1$ means there are $(K+1)$ packets in the system at the instant $t=(n+1)^{+}$; that is, the system is full. For this case, none of the packets in the system leaves, and there is at least one packet arrival; or one of the packets in the system leaves, and there are two packet (one SU packet and one PU packet) arrivals. So the transition probability matrix $\mathbf{Q}_{K, K+1}$ can be given as follows:

$$
\mathbf{Q}_{K, K+1}=\left(\begin{array}{cc}
\bar{\lambda}_{1} \gamma_{K} \bar{\mu}_{2} & \lambda_{1}\left(1-\left(1-\gamma_{K}\right) \mu_{2}\right) \\
0 & \gamma_{K}\left(\lambda_{1} \mu_{1}+\bar{\mu}_{1}\right)
\end{array}\right) .
$$

(5) At the instant $t=n^{+}$, if the system phase $u=K+1$, that is, there is no vacancy in the buffer, the system phase will be $v(v=K, K+1)$ at the instant $t=(n+1)^{+}$.

Similar to the matrix structure shown in (10), the transition probability matrix $\mathbf{Q}_{K+1, K}$ can be given as follows:

$$
\mathbf{Q}_{K+1, K}=\left(\begin{array}{ll}
\bar{\lambda}_{1}\left(1-\gamma_{K+1}\right) \mu_{2} & 0 \\
\bar{\lambda}_{1}\left(1-\gamma_{K+1}\right) \mu_{1} & 0
\end{array}\right) .
$$

The system phase $v=K+1$ means that there are $(K+1)$ packets in the system at the instant $t=(n+1)^{+}$; that is, the system is full. For this case, none of the packets in the system leaves, or one of the packets in the system leaves, and there is at least one packet arrival including also forced leaving of SU packet due to arrival of PU packet. So the transition probability matrix $\mathbf{Q}_{K+1, K+1}$ can be given as follows:

$$
\mathbf{Q}_{K+1, K+1}=\left(\begin{array}{cc}
\bar{\lambda}_{1}\left(\gamma_{K+1} \mu_{2}+\bar{\mu}_{2}\right) & \lambda_{1} \\
\bar{\lambda}_{1} \mu_{1} \gamma_{K+1} & \lambda_{1} \mu_{1}+\bar{\mu}_{1}
\end{array}\right) .
$$

The structure of the transition probability matrix $\mathbf{Q}$ indicates that the two-dimensional Markov chain $\left\{L_{n}, L_{n}^{(1)}\right\}$ is nonperiodic, irreducible, and positive recurrent [17]. Let $\pi_{i, j}$ be the steady-state distribution of the two-dimensional Markov chain, which can be given as follows:

$$
\pi_{i, j}=\lim _{n \rightarrow \infty} P\left\{L_{n}=i, L_{n}^{(1)}=j\right\} .
$$

Let $\Pi_{i}$ be the steady-state probability vector for the system being at phase $i . \Pi_{i}$ can be given as follows:

$$
\Pi_{i}= \begin{cases}\pi_{0,0}, & i=0, \\ \left(\pi_{i, 0}, \pi_{i, 1}\right), & 1 \leq i \leq K+1 .\end{cases}
$$

Combining the equilibrium equation and the normalization condition in the above Markov chain, we have

$$
\begin{gathered}
\left(\Pi_{0}, \Pi_{1}, \ldots, \Pi_{K}, \Pi_{K+1}\right) \mathbf{Q}=\left(\Pi_{0}, \Pi_{1}, \ldots, \Pi_{K}, \Pi_{K+1}\right), \\
\left(\Pi_{0}, \Pi_{1}, \ldots, \Pi_{K}, \Pi_{K+1}\right) \mathbf{e}=1,
\end{gathered}
$$

where $\mathbf{e}$ is a one's column vector.

Equation (21) is a linear system of equations with $2 \times(K+$ $1)+1$ unknowns. By using a Gaussian elimination method to solve the linear equations, we can obtain the steady-state distribution $\pi_{i, j}$ defined in (19).

\section{Performance Measures and Numerical Results}

4.1. Performance Measures. In this subsection, we give some performance measures for cognitive radio networks with an adaptive admission control scheme as follows.

We define the average system access rate $\theta$ of the SU packets as the average number of SU packets that are admitted to join the system per slot. In the adaptive admission control scheme proposed in this paper, the probability that a newly arriving SU packet is admitted to join the system is dependent on the Adaptive Factor and the total number of packets in the system at the arrival instant. So the average system access rate $\theta$ can be given as follows:

$$
\theta=\lambda_{2} \pi_{0,0}+\sum_{i=1}^{K+1} \frac{\lambda_{2}}{\alpha i+1}\left(\pi_{i, 0}+\pi_{i, 1}\right)
$$

We define the blocking rate $P_{B}$ of the SU packets as the average number of admitted SU packets that are blocked by the system per slot. An admitted SU packet will be blocked by the system in three cases: (1) Suppose that the channel is occupied by an SU packet and the buffer of the SU packets is already full in the previous slot. At current slot, the SU packet occupying the channel is not transmitted completely; or the SU packet occupying the channel is transmitted successfully, but a PU packet joins the system and occupies the channel. (2) Suppose that the channel is occupied by a PU packet, and the buffer of the SU packets is already full in the previous slot. At current slot, the PU packet occupying the channel is not transmitted completely; or the PU packet occupying the channel is transmitted successfully, but a new PU packet joins the system and occupies the channel. (3) Suppose that the channel is occupied by an SU packet, and there is only one vacancy in the buffer of the SU packets in the previous slot. At current slot, the SU packet occupying the channel is not transmitted completely and is interrupted by a newly arriving PU packet. The interrupted SU packet returns back to the buffer and occupies the only one vacancy. Therefore, the blocking rate $P_{B}$ of the SU packets can be given as follows:

$$
\begin{aligned}
P_{B}= & \frac{\lambda_{2}}{\alpha(K+1)+1} \\
& \times\left(\left(\bar{\mu}_{2}+\lambda_{1} \mu_{2}\right) \pi_{K+1,0}+\left(\bar{\mu}_{1}+\lambda_{1} \mu_{1}\right) \pi_{K+1,1}\right) \\
& +\frac{\lambda_{2}}{\alpha K+1} \lambda_{1} \bar{\mu}_{2} \pi_{K, 0} .
\end{aligned}
$$

We define the throughput $S$ of the SU packets as the average number of SU packets transmitted successfully per slot. An SU packet can be transmitted successfully if and only if this SU packet is admitted to join the system, not blocked by the system, and not forced to leave the system before the transmission is completely finished. The blocking rate of the SU packets can be obtained in (23). When a PU packet arrives at the system during the transmission time of an SU packet, if the buffer of the SUs is full, the SU packet queueing at the 
TABLE 1: Common parameters setting in the numerical results.

\begin{tabular}{lc}
\hline Parameter & Value \\
\hline Slot & $1 \mathrm{~ms}$ \\
Average packet size & 2750 Bytes \\
Data rate in physical layer & $11 \mathrm{Mbps}$ \\
\hline
\end{tabular}

end of the buffer will be forced to leave the system. Therefore, the throughput $S$ of the $S U$ packets is given as follows:

$$
S=\theta-P_{B}-\lambda_{1} \bar{\mu}_{2} \pi_{K+1,0} .
$$

We define the latency of an SU packet as the time period from the instant an SU packet is admitted to join the system to the instant that the SU packet is successfully transmitted. In fact, the latency of an SU packet is the sojourn time of that SU packet.

Let $L_{n}^{(2)}$ be the number of SU packets in the system at the instant $t=n^{+}$, and let $L^{(2)}=\lim _{n \rightarrow \infty} L_{n}^{(2)}$ be the number of SU packets in steady state. We can get the average number $E\left[L^{(2)}\right]$ of the $S \mathrm{U}$ packets as follows:

$$
E\left[L^{(2)}\right]=\sum_{i=0}^{K+1} i P\left\{L^{(2)}=i\right\}=\sum_{i=1}^{K+1} i \pi_{i, 0}+\sum_{i=0}^{K} i \pi_{i+1,1} .
$$

By using Little's law [19], the average latency $\delta$ of the SU packets can be given as follows:

$$
\delta=\frac{E\left[L^{(2)}\right]}{S} .
$$

4.2. Numerical Results. In the numerical results, we consider the $2.4 \mathrm{GHZ}$ spectrum band in Wi-Fi based wireless networks. Following the IEEE $802.11 \mathrm{~b} / \mathrm{g}$ standard and referencing the parameter setting in [7], the common parameters used in the numerical results are summarized in Table 1.

Based on Table 1, the transmission rate for the packets can be calculated as $\mu_{1}=\mu_{2}=0.5$.

Moreover, the data set for the Adaptive Factor is set as $\alpha=$ $\{0.0,0.1, \ldots, 0.9,1.0\}$, in which the case of $\alpha=0$ indicates the conventional system access strategy without any admission control. In this way, the influence of the Adaptive Factor $\alpha$ on the system performance and on the efficiency of the proposed adaptive admission control scheme can be shown.

At the same time, in order to distinctively investigate the influences of the arrivals for the PU packets and the SU packets on the performance measures, we set the arrival rates of the PU packets and the SU packets as $\lambda_{1}=\{0.2,0.3\}$ and $\lambda_{2}=\{0.4,0.6\}$. Additionally, as an example, we assume the buffer capacity of the SU is fixed at $K=10$. We remark here that with a larger buffer capacity of the SUs, both the throughput and the average latency of the SU packets will be higher.

Figure 2 shows how the throughput $S$ of the SU packets changes with respect to the Adaptive Factor $\alpha$ for the different arrival rates.

From Figure 2, we observe that, for the same arrival rate $\lambda_{1}$ of the PU packets and the same arrival rate $\lambda_{2}$ of the SU

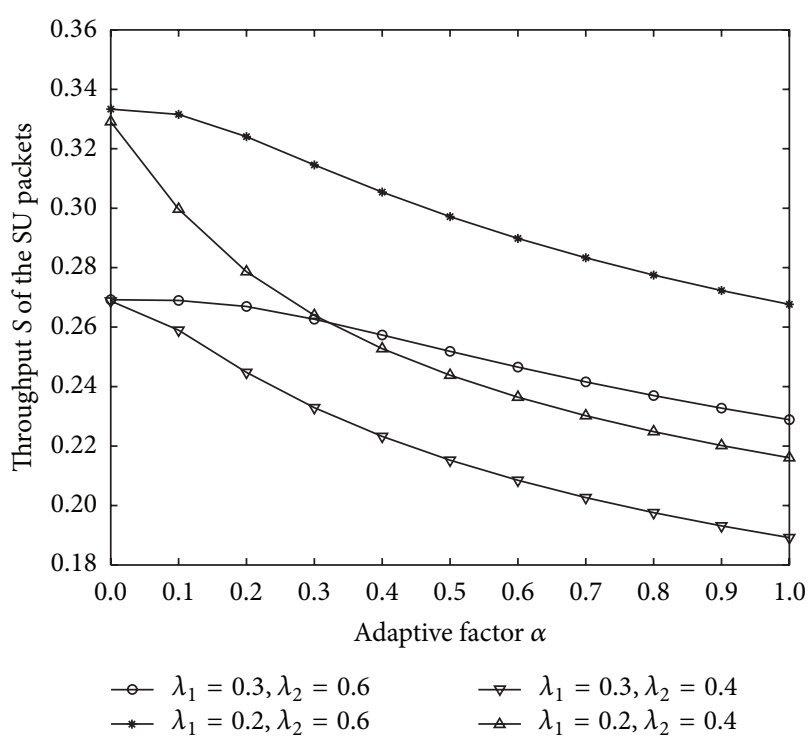

FIgURE 2: Throughput $S$ of the SU packets versus Adaptive Factor $\alpha$.

packets, the throughput $S$ of the SU packets will decrease as the Adaptive Factor $\alpha$ increases. The reason is that the larger the Adaptive Factor is, the less likely it is that a newly arriving SU packet will be admitted to join the system, so the throughput of the SU packets will be lower.

On the other hand, from Figure 2, we see that for the same arrival rate $\lambda_{1}$ of the PU packets and the same Adaptive Factor $\alpha$, the throughput $S$ of the SU packets will increase as the arrival rate $\lambda_{2}$ of the $S U$ packets increases. The reason is that the larger the arrival rate of the SU packets is, the more SU packets can join the system, so the throughput of the SU packets will be lower.

Moreover, we find that for the same arrival rate $\lambda_{2}$ of the SU packets and the same Adaptive Factor $\alpha$, the larger the arrival rate $\lambda_{1}$ of the $P U$ packets is, the smaller the throughput $S$ of the SU packets will be. This is because as the arrival rate of the PU packets increases, the possibility that the channel is occupied by a PU packet is higher; then the possibility for an SU packet occupying the channel will be lower. On the other hand, the possibility for an SU packet being interrupted by $\mathrm{PU}$ packets will be higher. As a result, the throughput of the SU packets will decrease.

We examine the influence of the Adaptive Factor $\alpha$ on the average latency $\delta$ of the SU packets for the different arrival rates in Figure 3.

In Figure 3, we conclude that for the same arrival rate $\lambda_{1}$ of the PU packets and the same arrival rate $\lambda_{2}$ of the SU packets, the average latency $\delta$ of the SU packets will decrease as the Adaptive Factor $\alpha$ increases. The reason is that the larger the Adaptive Factor is, the lower the possibility that a newly arriving SU packet will be admitted to join the system is, the less the number of SU packets in the system will be, and this will result in a decrease in the average latency of the SU packets.

On the other hand, from Figure 3, we find that for the same arrival rate $\lambda_{1}$ of the PU packets and the same Adaptive 


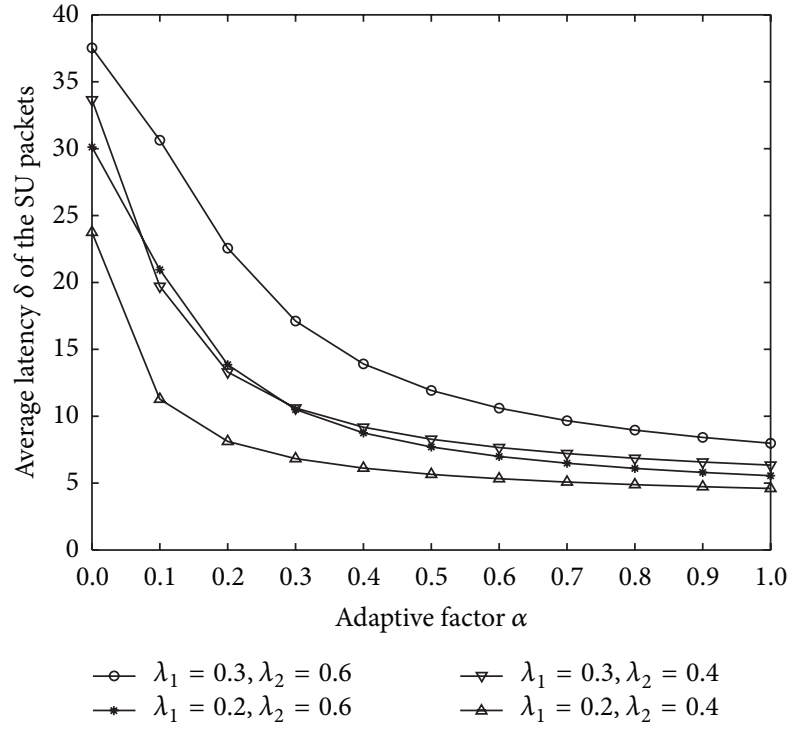

FIGURE 3: Average latency $\delta$ of the SU packets versus Adaptive Factor $\alpha$.

Factor $\alpha$, the average latency $\delta$ of the SU packets will increase as the arrival rate $\lambda_{2}$ of the SU packets increases. The reason is that the larger the arrival rate of the SU packets is, the more SU packets can join the system and wait in the buffer, and this will induce an increase in the average latency of the SU packets.

Moreover, we see that for the same arrival rate $\lambda_{2}$ of the SU packets and the same Adaptive Factor $\alpha$, the larger the arrival rate $\lambda_{1}$ of the PU packets is, the longer the average latency $\delta$ of the SU packets will be. This is because as the arrival rate of the PU packets increases, the possibility that the channel is occupied by a PU packet will increase, so the time length for an SU packet waiting in the buffer will increase. This will make the average latency of the SU packets increase.

When the Adaptive Factor is set to be $\alpha=0$ in Figures 2 and 3, we can obtain the system performance of the conventional system access strategy without any admission control. In the adaptive admission control scheme we can conclude from the trends of the performance measures of the SU packets, that the average latency of the SU packets is reduced significantly, while the throughput of the SU packets will be decreased slightly.

Additionally, from Figures 2 and 3, we know that as the Adaptive Factor $\alpha$ increases and the average latency $\delta$ of the SU packets will decrease, but also the throughput $S$ of the SU packets will decrease. So we conclude that there is a trade-off when setting the Adaptive Factor $\alpha$. In order to formulate the joint optimal problem of throughput $S$ and average latency $\delta$ of the SU packets, we construct a net benefit function $F(\alpha)$ as follows:

$$
F(\alpha)=C_{1} S-C_{2} \delta
$$

where $C_{1}$ and $C_{2}$ are supposed to be the reward of the throughput $S$ and the cost of the average latency $\delta$ of the SU packets, respectively.

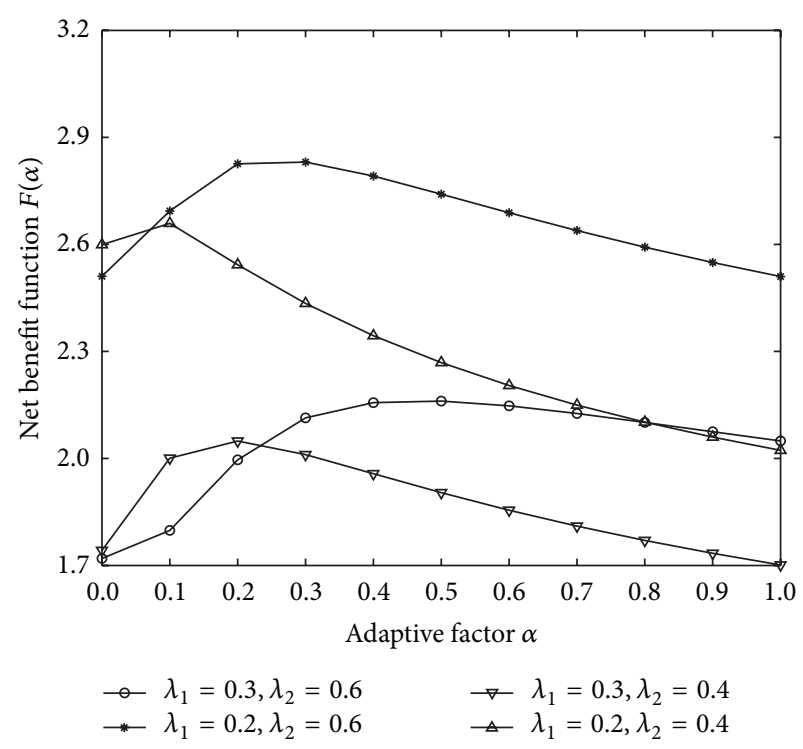

Figure 4: Net benefit function $F(\alpha)$ versus Adaptive Factor $\alpha$.

From (27), the optimal Adaptive Factor $\alpha^{*}$ can be given as follows:

$$
\alpha^{*}=\arg \max \{F(\alpha)\}
$$

where "arg max" stands for the argument of the maximum [20].

By setting $C_{1}=10$ and $C_{2}=0.03$ as an example, we plot how the net benefit function $F(\alpha)$ changes with respect to the Adaptive Factor $\alpha$ in Figure 4 .

As expected we can see in Figure 4, that there is a maximum net benefit when the Adaptive Factor is set to the optimal value for all the arrival rates of packets. For example, when $\lambda_{1}=0.3, \lambda_{2}=0.6$, the optimal Adaptive Factor is $\alpha^{*}=0.5$ and the maximum net benefit is $F\left(\alpha^{*}\right)=2.16$; when $\lambda_{1}=0.2, \lambda_{2}=0.6$, the optimal Adaptive Factor is $\alpha^{*}=0.3$ and the maximum net benefit is $F\left(\alpha^{*}\right)=2.83$; when $\lambda_{1}=0.3, \lambda_{2}=0.4$, the optimal Adaptive Factor is $\alpha^{*}=0.2$ and the maximum net benefit is $F\left(\alpha^{*}\right)=2.05$; when $\lambda_{1}=0.2, \lambda_{2}=0.4$, the optimal Adaptive Factor is $\alpha^{*}=0.1$ and the maximum net benefit is $F\left(\alpha^{*}\right)=2.66$.

\section{Performance Optimization}

In the adaptive admission control scheme proposed in this paper, when an SU packet arrives at the system, this SU packet may be not admitted by the system. Even this SU packet is admitted to join the system; it may be blocked by the system. In other word, the transmission for an SU packet is not guaranteed. From the view point of the SU packets, it is necessary to make optimization for their actions to obtain the maximum benefit. So, in this section, we firstly give some assumptions, and then we compare the individually optimal strategy and the socially optimal strategy for the SU packets. At last, in order to coordinate the two optimal strategies, we propose a pricing mechanism. 
5.1. Assumptions for Performance Optimization. In this subsection, we give some assumptions that will be used in the following optimizations.

(1) We assume that a newly arriving SU packet is not aware the number of packets already in the system and does not know whether to be admitted by the system before making any decisions. This assumption is different from the observable queue case in [14]. Additionally, an SU packet will either irrevocably join the system or not join the system at all.

(2) Let $R$ be the reward for a successful transmission of an SU packet. Since the admission to the system for the SU packets is not guaranteed, we refer the arrival of a new SU packet as a trial. There is a cost $T(T<R)$ associated with each trial. That is to say, when a new SU packet arrives at the system, it will pay a cost $T$ for trying to join the system, no matter whether or not it will be transmitted successfully.

(3) We denote the potential arrival rate of the SU packets as $\Lambda$.

5.2. Comparison between Individually and Socially Optimal Strategies. We firstly discuss the individually optimal strategy [20] for the SUs. From the view point of a single SU packet considered in this paper, there is a mixed trial strategy described with a fraction $q, 0 \leq q \leq 1$, which is the probability for an SU packet trying to join the system. We denote the individually optimal trial rate as $\lambda_{e}$ and the individually optimal trial probability as $q_{e}$. Then we have $\lambda_{e}=q_{e} \Lambda$, where $\Lambda$ is the potential arrival rate of the SU packets.

Both of the optimal trial probability $q_{e}$ and the actual joining rate $\gamma_{i}$ introduced in Section 2 will impact the accessibility of the SU packets. With the optimal trial probability $q_{e}$, an SU packet will reach Nash equilibrium [20]. With the actual joining rate $\gamma_{i}$ introduced in the admission control scheme, the access of the SU packets can be controlled adaptively based on the number of packets already in the system.

We can obtain the expression for the probability $\varepsilon\left(\lambda_{2}\right)$ that an SU packet can be successfully transmitted as follows:

$$
\varepsilon\left(\lambda_{2}\right)=\frac{S}{\lambda_{2}},
$$

where $S$ is the throughput of the SU packets given in (24), and $\lambda_{2}\left(0<\lambda_{2}<1\right)$ is the arrival rate of the SU packets.

The individual net benefit $B_{I}\left(\lambda_{2}\right)$ for an SU packet who tries to join the system is given by

$$
B_{I}\left(\lambda_{2}\right)=\varepsilon\left(\lambda_{2}\right)(R-T)-\left(1-\varepsilon\left(\lambda_{2}\right)\right) T=\varepsilon\left(\lambda_{2}\right) R-T .
$$

Considering the complexity of the individual net benefit function, we explore the monotonic property of $B_{I}\left(\lambda_{2}\right)$ in (30) with numerical results. Taking the parameters used in Figures 2 and 3 and setting $R=60, T=35, \lambda_{1}=0.3, \lambda_{2} \in(0.01,0.50]$ as an example, we show how the individual net benefit $B_{I}\left(\lambda_{2}\right)$ changes with respect to the arrival rate $\lambda_{2}$ of the SU packets for the different Adaptive Factors $\alpha$ in Figure 5.

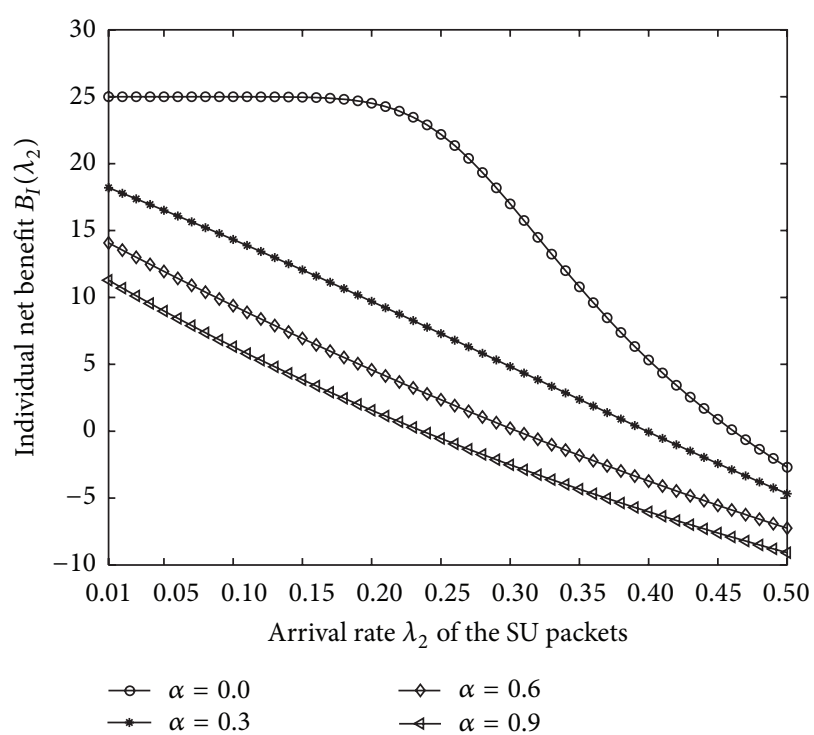

FIGURE 5: Dependency of the individual net benefit $B_{I}\left(\lambda_{2}\right)$ on $\lambda_{2}$.

As shown in Figure 5, the individual net benefit for an SU packet is monotonely decreasing as the arrival rate of the SU packets increases. We consider three cases for the optimal trial strategy of a single SU packet as follows:

(1) $B_{I}\left(0^{+}\right) \leq 0$. In this case, even if no other SU packets join the system, the SU packet who tries to join the system will not get a positive benefit. So, the trial strategy with probability $q_{e}=0$ is an optimal strategy and no other optimal strategy is possible.

(2) $B_{I}(\Lambda) \geq 0$. In this case, even if all the potential arrival SU packets try to join the system, all of them will get nonnegative benefits. So, the trial strategy with probability $q_{e}=1$ is an optimal strategy and no other optimal strategy is possible.

(3) $B_{I}\left(0^{+}\right)>0$ and $B_{I}(\Lambda)<0$. In this case, if all the SU packets join the system with probability $q=1$, the $\mathrm{SU}$ packet who tries to join the system will get a negative net benefit. So $q=1$ is not an optimal strategy. On the other hand, if all the SU packets join the system with probability $q=0$, the SU packet who tries to join the system will get a positive net benefit. So $q=0$ is not an optimal strategy too. Therefore, there is an optimal trial probability $q_{e}=\lambda_{e} / \Lambda$, where $\lambda_{e}$ can be obtained by solving the equation $B_{I}\left(\lambda_{e}\right)=0$.

Thereupon, we discuss the socially optimal strategy [20]. The social net benefit $B_{S}\left(\lambda_{2}\right)$ is defined as follows:

$$
B_{S}\left(\lambda_{2}\right)=\lambda_{2}\left(\varepsilon\left(\lambda_{2}\right) R-T\right) .
$$

We also explore the monotonic property of $B_{S}\left(\lambda_{2}\right)$ in (31) with numerical results. By applying the same parameters as used in Figure 5, we show how the social net benefit $B_{S}\left(\lambda_{2}\right)$ changes with respect to the arrival rate $\lambda_{2}$ of the SU packets for the different Adaptive Factors $\alpha$ in Figure 6.

From Figure 6, we conclude that as the arrival rate of the SU packets increases, the function of the social net benefit 


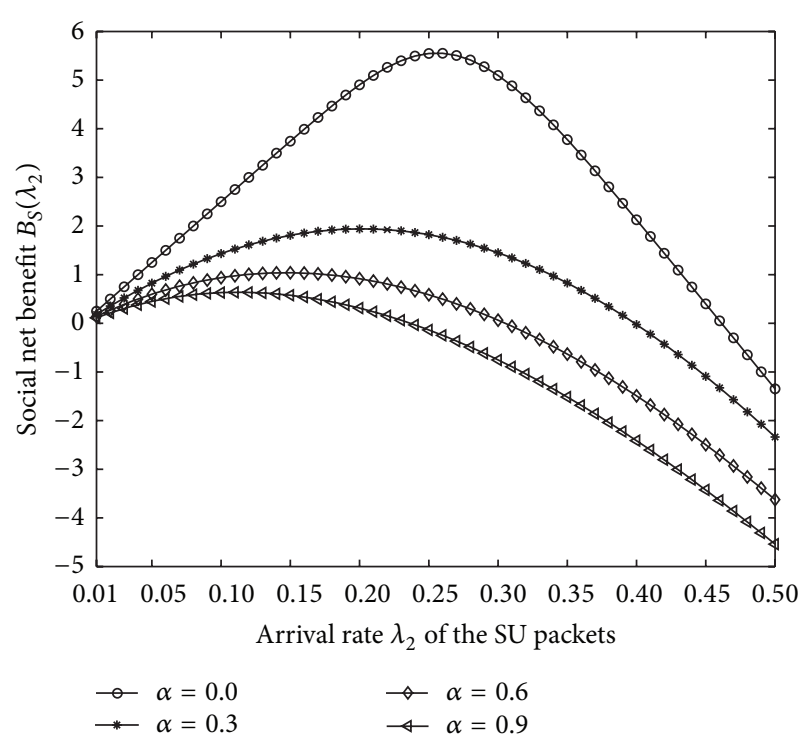

Figure 6: Function $B_{S}\left(\lambda_{2}\right)$ of the social net benefit.

TABLE 2: Numerical results for the individually and socially optimal strategies.

\begin{tabular}{lcccccc}
\hline$\alpha$ & \multicolumn{2}{c}{$\lambda_{e}$} & \multicolumn{2}{c}{$q_{e}$} & $\lambda^{*}$ & $q^{*}$ \\
& $\min$ & $\max$ & $\min$ & $\max$ & & \\
\hline 0.00 & 0.46 & 0.47 & 0.92 & 0.94 & 0.26 & 0.52 \\
0.30 & 0.39 & 0.40 & 0.78 & 0.80 & 0.20 & 0.40 \\
0.60 & 0.30 & 0.31 & 0.60 & 0.62 & 0.15 & 0.30 \\
0.90 & 0.23 & 0.24 & 0.46 & 0.48 & 0.11 & 0.22 \\
\hline
\end{tabular}

shows an upper convex behavior. Hence, the socially optimal trial rate $\lambda^{*}$ can be given as follows:

$$
\lambda^{*}=\underset{0 \leq \lambda_{2} \leq \Lambda}{\arg \max }\left\{B_{S}\left(\lambda_{2}\right)\right\} .
$$

Then we can get the socially optimal trial probability $q^{*}$ as follows:

$$
\begin{gathered}
q^{*}=1, \quad \lambda^{*} \geq \Lambda, \\
q^{*}=\frac{\lambda^{*}}{\Lambda}, \quad 0<\lambda^{*}<\Lambda .
\end{gathered}
$$

By setting the potential arrival rate for the SU packets as $\Lambda=0.5$ in Figures 5 and 6 , for the different Adaptive Factors $\alpha$, we obtain the value ranges of the individually optimal trial rate $\lambda_{e}$ and the values of the socially optimal trail rate $\lambda^{*}$. We can also calculate the value ranges of the individually optimal trial probability $q_{e}$ with $q_{e}=\lambda_{e} / \Lambda$ and the values of the socially optimal trail probability $q^{*}$ with $q^{*}=\lambda^{*} / \Lambda$. We summarize these numerical results in Table 2.

In Table 2, the estimates for different numerical results are accurate to two decimal places.

From Table 2, we find that, for all the Adaptive Factors $\alpha$, the socially optimal trail rate $\lambda^{*}$ is smaller than the individually optimal trial rate $\lambda_{e}$, and the socially optimal trial probability $q^{*}$ is smaller than the individually optimal trial probability $q_{e}$. These conclusions are consistent with the results given in $[14,20]$.

On the other hand, we also observe that as the Adaptive Factor $\alpha$ increases, both of the optimal trail rates and the optimal trial probabilities show a decreasing trend. The reason is that the larger the Adaptive Factor is, a newly arriving SU packet is less likely to be admitted by the system. In order to reduce the trial cost of the refused SU packets, the optimal trail rate and the optimal trial probability will be lower.

5.3. Pricing Mechanism. In order to oblige the single SU packet to adopt socially optimal strategy, we give a pricing mechanism by subtracting an extra fee $f$ from the reward for the SU packet with successful transmission. When the extra fee $f$ is imposed, the net benefit $B_{T}\left(\lambda_{2}\right)$ for an SU packet who tries to join the system can be given as follows:

$$
B_{T}\left(\lambda_{2}\right)=\varepsilon\left(\lambda_{2}\right)(R-f)-T .
$$

By setting $\lambda_{2}=\lambda^{*}$ in (34), we can obtain the net benefit $B_{T}\left(\lambda^{*}\right)$ as follows:

$$
B_{T}\left(\lambda^{*}\right)=\varepsilon\left(\lambda^{*}\right)(R-f)-T .
$$

By solving $B_{T}\left(\lambda^{*}\right)=0$, the extra fee $f$ can be given as follows:

$$
f=R-\frac{T}{\varepsilon\left(\lambda^{*}\right)} .
$$

Specially, in the case of $\lambda^{*}=\Lambda$, the extra fee $f$ will be equal to or less than $(R-T / \varepsilon(\Lambda))$.

For example, by using the numerical results given in Table 2, we can calculate the extra fee $f$ with (36) for different Adaptive Factors $\alpha$ as follows: when the adaptive Factor $\alpha$ is 0 , the extra fee $f$ is 26.45; when the adaptive Factor $\alpha$ is 0.30 , the extra fee $f$ is 13.03; when the adaptive Factor $\alpha$ is 0.60 , the extra fee $f$ is 9.92; when the adaptive Factor $\alpha$ is 0.90 , the extra fee $f$ is 8.53 .

Conclusively, as the Adaptive Factor $\alpha$ increases, the extra fee $f$ shows a decreasing trend. The reason is that as the Adaptive Factor increases, the gap between the individually and the socially optimal trail rate shown in Table 2 will decrease, so the extra fee $f$ will be reduced accordingly.

\section{Conclusions}

In order to reduce the SU packets' greater latency due to larger number of SU packets access to the system without any restrictions, in this paper, we proposed an adaptive admission control scheme for the SUs in cognitive radio networks. We introduced an Adaptive Factor into the admission control scheme so as to control the system access behavior of the SU packets adaptively. The system access probability of a newly arriving SU packet is determined by the Adaptive Factor and the total number of packets already in the system at the arriving instant. Based on the working principle of the adaptive admission control scheme and the priority of the PUs in cognitive radio networks, we built a discrete-time 
preemptive queueing model with priority and adjustable joining rate. We constructed a two-dimensional Markov chain and gave the transition probability matrix of the Markov chain to exactly analyze the queueing model. Accordingly, we derived the formulas for the different performance measures. Moreover, we gave the individually optimal strategy and the socially optimal strategy for the SU packets, and then a pricing mechanism was presented to coordinate the two optimal strategies.

In this paper, we assumed the interarrival and transmission times for the packets to follow geometric distributions [14]. As a future work, we will extend the system model by considering some nongeometric distributions for the interarrivals and the transmission times of the packets.

\section{Acknowledgments}

This work was supported in part by Hebei Province Science Foundation (no. F2012203093), China, and was supported in part by MEXT, Japan.

\section{References}

[1] Q. Zhao and B. M. Sadler, "A survey of dynamic spectrum access," IEEE Signal Processing Magazine, vol. 24, no. 3, pp. 7989, 2007.

[2] B. Wang and K. J. R. Liu, "Advances in cognitive radio networks: a survey," IEEE Journal of Selected Topics in Signal Processing, vol. 5, no. 1, pp. 5-23, 2011.

[3] A. De Domenico, E. C. Strinati, and M.-G. Di Benedetto, "A survey on MAC strategies for cognitive radio networks," IEEE Communications Surveys and Tutorials, vol. 14, no. 1, pp. 21-44, 2012.

[4] P. Yadav, S. Chaterjee, and P. P. Bhattacharya, "A survey on dynamic spectrum access techniques in cognitive radio," International Journal of Next-Generation Networks, vol. 4, no. 4, pp. 27-46, 2012.

[5] J. Marinho and E. Monteiro, "Cognitive radio: survey on communication protocols, spectrum decision issues, and future research directions," Wireless Networks, vol. 18, no. 2, pp. 147164, 2012.

[6] H. Su and X. Zhang, "Cross-layer based opportunistic MAC protocols for QoS provisionings over cognitive radio wireless networks," IEEE Journal on Selected Areas in Communications, vol. 26, no. 1, pp. 118-129, 2008.

[7] K. J. Kim, K. S. Kwak, and B. D. Choi, "Performance analysis of opportunistic spectrum access protocol for multi-channel cognitive radio networks," Journal of Communications and Networks, vol. 15, no. 1, pp. 77-86, 2013.

[8] S. De Clercq, B. Steyaert, and H. Bruneel, "Queue content analysis in a 2-class discrete-time queueing system under the slot-bound priority service rule," Mathematical Problems in Engineering, vol. 2012, Article ID 425630, 20 pages, 2012.

[9] C. T. Do, N. H. Tran, and C. S. Hong, "Throughput maximization for the secondary user over multi-channel cognitive radio networks," in Proceedings of the International Conference on Information Networking, pp. 65-69, Bangkok, Thailand, February 2012.

[10] P. G. Smith, A. Firag, P. A. Dmochowski, and M. Shafi, "Analysis of the $\mathrm{M} / \mathrm{M} / \mathrm{N} / \mathrm{N}$ queue with two types of arrival process: applications to future mobile radio systems," Journal of Applied Mathematics, vol. 2012, Article ID 123808, 14 pages, 2012.

[11] Y. Wang, H. R. Karimi, and Z. Xiang, " $H_{\infty}$ Control for networked control systems with time delays and packet dropouts," Mathematical Problems in Engineering, vol. 2013, Article ID 635941, 10 pages, 2013.

[12] J. Hu, J. Liang, H. R. Karimi, and J. Cao, "Sliding intermittent control for BAM neural networks with delays," Abstract and Applied Analysis, vol. 2013, Article ID 615947, 15 pages, 2013.

[13] S. Havedanloo and H. R. Karimi, "Improving the performance metric of wireless sensor networks with clustering markov chain model and multilevel fusion," Mathematical Problems in Engineering, vol. 2013, Article ID 783543, 11 pages, 2013.

[14] H. Li and Z. Han, "Socially optimal queuing control in cognitive radio networks subject to service interruptions: to queue or not to queue?" IEEE Transactions on Wireless Communications, vol. 10, no. 5, pp. 1656-1666, 2011.

[15] A. Turhan, M. Alanyali, and D. Starobinski, "Optimal admission control of secondary users in preemptive cognitive radio networks," in Proceedings of the International Symposium on Modeling and Optimization in Mobile, Ad Hoc and Wireless Networks, pp. 138-144, Paderborn, Germany, May 2012.

[16] A. S. Alfa, Queueing Theory for Telecommunications: Discrete Time Modelling of a Single Node System, Springer, 2010.

[17] H. Takagi, Queueing Analysis, Volume 3: Discrete-Time Systems, North-Holland, 1993.

[18] Y. Liu, H. R. Karimi, and X. Liu, "Distributed consensus for discrete-time directed networks of multiagents with timedelays and random communication links," Abstract and Applied Analysis, vol. 2013, Article ID 158731, 9 pages, 2013.

[19] N. Tian and Z. G. Zhang, Vacation Queueing Models: Theory and Applications, Springer, 2006.

[20] R. Hassin and M. Haviv, To Queue or Not to Queue: Equilibrium Behaviour in Queueing Systems, Kluwer Academic Publishers, 2003. 


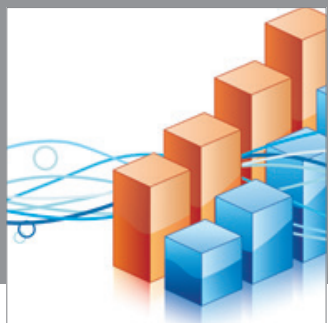

Advances in

Operations Research

mansans

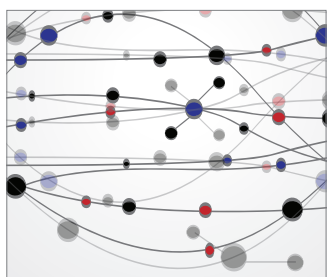

The Scientific World Journal
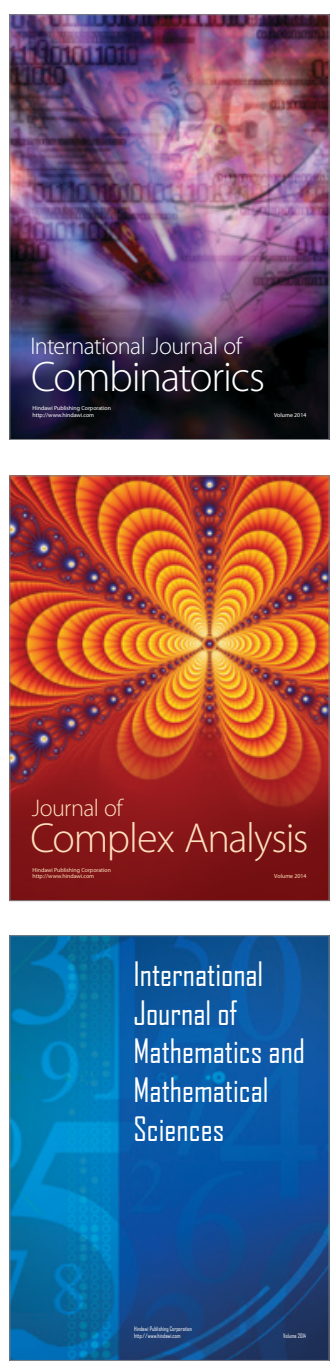
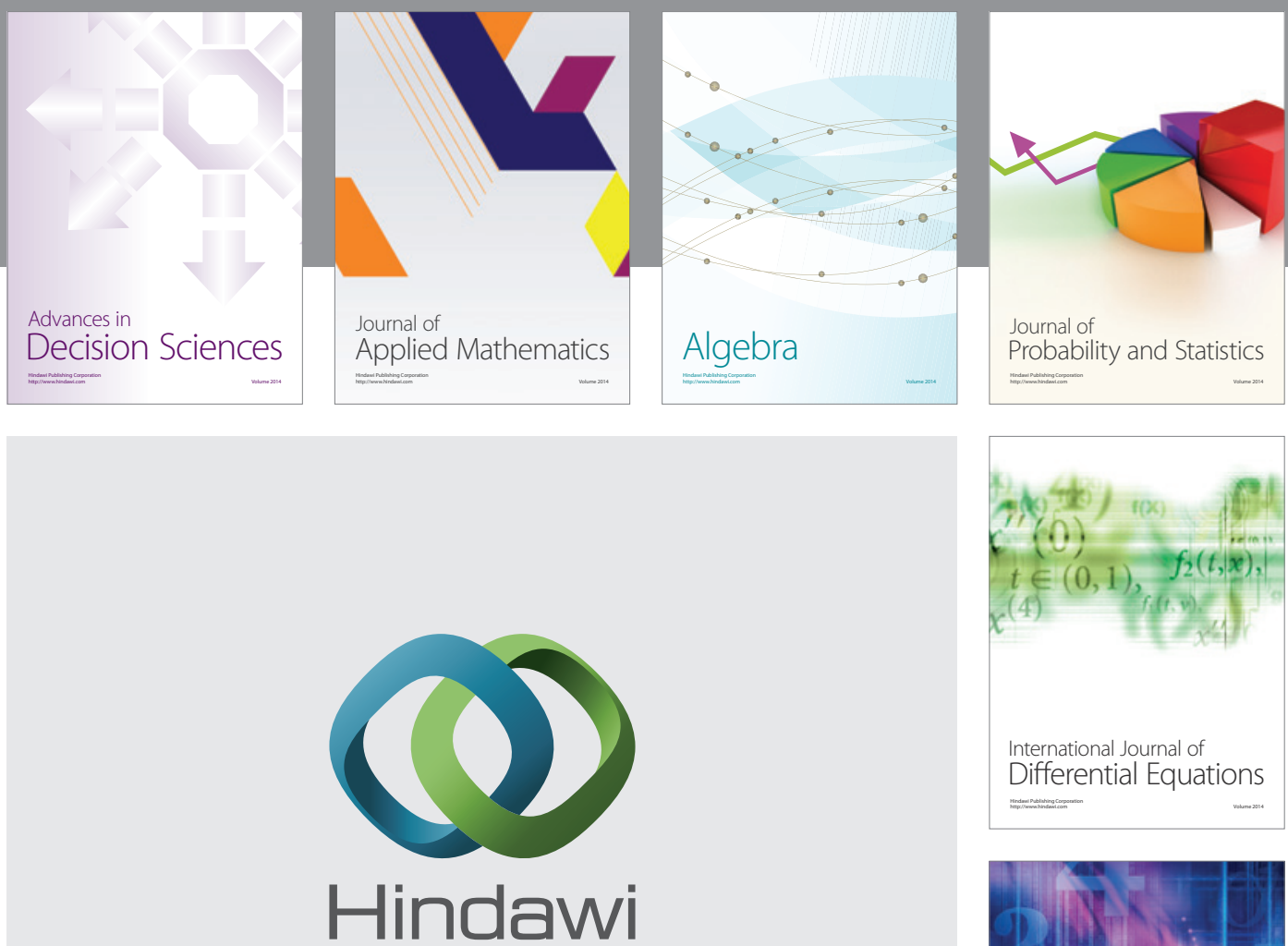

Submit your manuscripts at http://www.hindawi.com
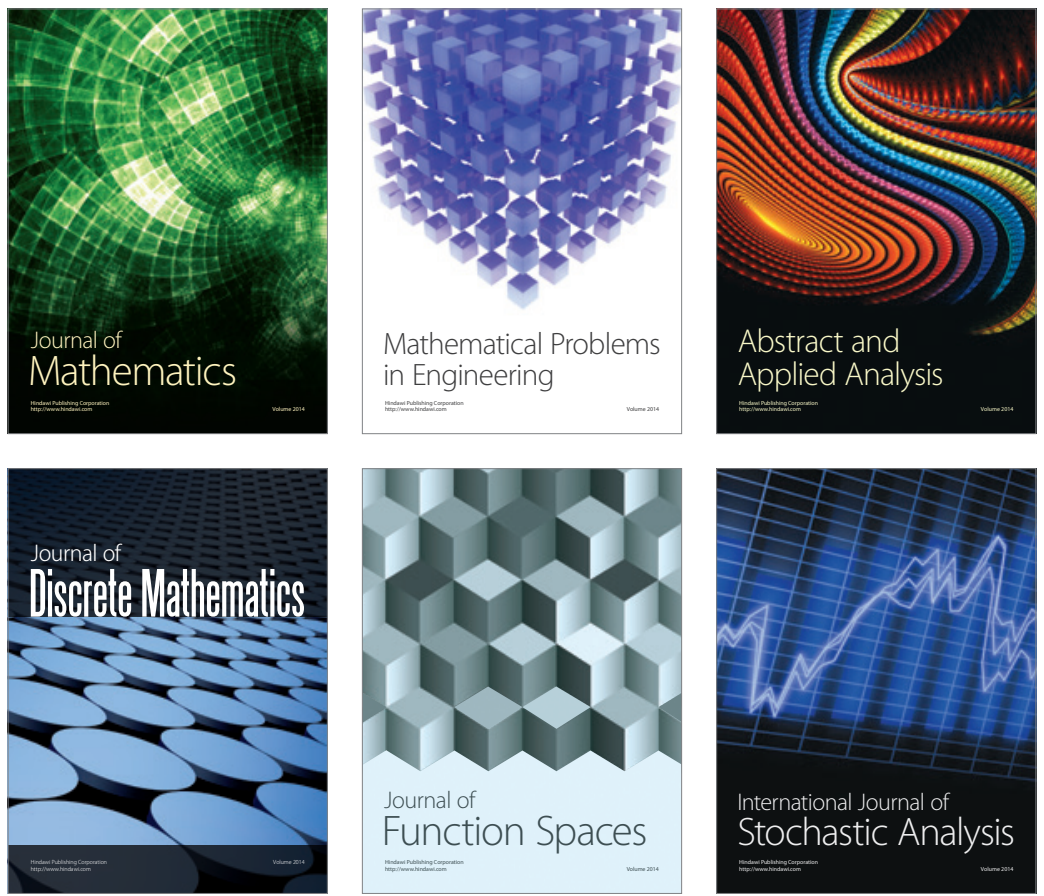

Journal of

Function Spaces

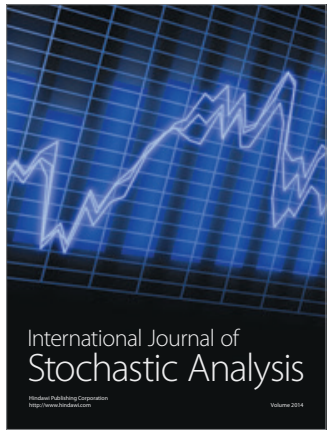

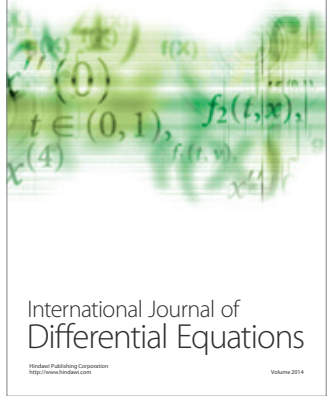
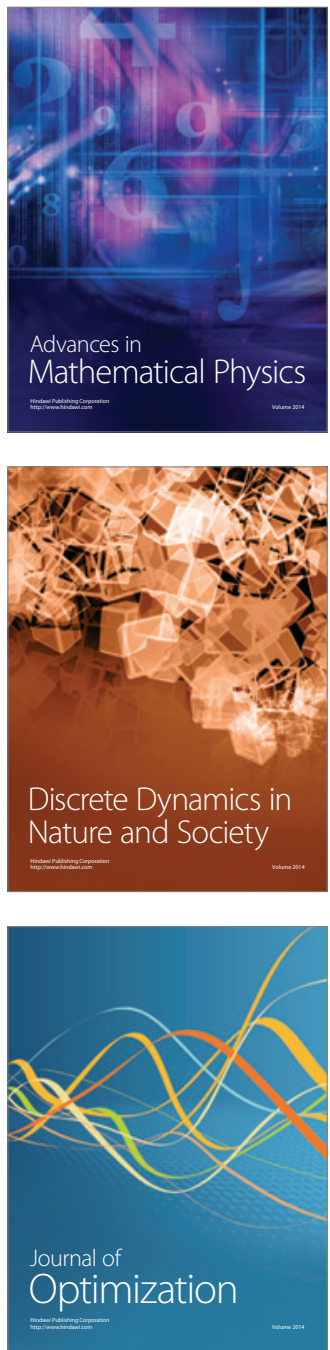